\title{
The assessment of discriminative encoding in memory for paired associates*
}

\author{
WILLARD N. RUNQUIST \\ University of Alberta, Edmonton, Alberta, Canada
}

\begin{abstract}
A technique for providing for the measurement of encoding stability and intralist stimulus discrimination is presented and applied to the analysis of some paired-associate data. Essentially, the measure involves a recall test for all stimuli in the list except one, then presenting either a previously tested stimulus or the missing stimulus. Recognition of repeated stimuli reflects code stability, and recognition of the missing stimulus reflects stimulus discrimination.
\end{abstract}

Consider two paired-associates $A-B$ and $C-D$ being acquired more or less simultaneously. After some learning has taken place, the presentation of $\mathrm{A}$ will sometimes produce $D$ and recallability of $B$ is poorer than if A-B were acquired alone.

How does this interaction take place? One model is the familiar doctrine of competing associations. Presentation of $\mathrm{A}$ results in the simultaneous activation of both $\mathrm{B}$ and $\mathrm{D}$ so that the occurrence of $\mathrm{D}$ blocks or replaces $B$.

A second model states that there are two independent associations, A-B and C-D, and that interference occurs due to the failure of the learner to discriminate between $A$ and $C$. That is, the presentation of $A$ will evoke $B$ when $A$ is correctly identified or encoded as $A$, but will evoke $\mathrm{D}$ when it is incorrectly encoded as if it were $\mathrm{C}$.

The critical difference is the source of interaction among traces. In the former, the to-be-recalled (target) items interact directly. We shall call this associative interference. In the other case, the interaction occurs only at the level of stimulus encoding. That is, there is no direct interaction among associative traces at all, merely confusion among stimuli: cue confusion.

The issue addressed in this paper is the measure of cue confusion, or conversely the extent of cue discrimination. When investigators talk about stimulus discriminability, they typically are referring to perceptual discriminability. Thus, one approach has been to assess initial discriminability of stimuli, then relate learning performance to discriminability. For example, Rothkopf (1957) had Ss make same-different judgments on Morse code signals and then related confusion errors in the scaling task to learning performance on sets of these stimuli varying in confusability. The most notable limitation to this approach is that the performance during learning reflects not only changes in discriminability, but other processes involving association, etc.

\footnotetext{
*Supported by Research Grant AP-88 from the National Research Council of Canada. Marva Blackmore supervised the data collection. Es were Marion Nicely and Linda Hughes.
}

While it is theoretically feasible to obtain discrimination measures at various stages during learning, there are a number of technical problems which limit this approach. Except with certain classes of nonverbal stimuli, perceptual confusions can usually be obtained only under conditions involving severe signal deprivation, such as tachistoscopic exposure, or by embedding the stimulus in a noisy background. These conditions are hardly typical of the conditions under which the $S$ functions in most learning experiments, where perceptual difficulties are usually minimal, and yet similarity among stimuli is still a factor.

More recently, Martin (1968) proposed that the accuracy with which an $S$ can identify a given stimulus as coming from the list being learned provides an index of stimulus encoding. The test has been used with both verbal (Martin, 1967) and nonverbal stimuli (Ellis \& Muller, 1964) and will result in recognition errors under the learning conditions imposed in most learning experiments. While this test can provide information about the stimulus codes being used, they are not necessarily relevant as measures of intralist stimulus discrimination. These tests require the $S$ to discriminate between list stimuli and stimuli not in the list. If the list stimuli contain redundant attributes and the $S$ ignores those attributes, he may be able to discriminate perfectly among the list stimuli but fail to discriminate between list and extralist stimuli when the latter consist of coded attributes with redundant attributes changed (Runquist \& Evans, 1972).

The problem, then, is to assess the discrimination among the stimuli comprising the particular list being learned. Logically, such a measure of cue confusion should have several properties: (1) It should be independent of the successful recall of the associated response term. (2) It should require the comparison of the test stimulus with the memorial representation of other stimuli from within the list, as this represents the task demands involved in learning. (3) Any encoding activities should be the same as those used in the retrieval process. 
Table 1

Possible Outcomes on Recognition Test

\begin{tabular}{|c|c|c|c|c|c|c|c|c|c|c|}
\hline Recall Code & $\mathrm{a}$ & $\mathrm{a}$ & $\mathbf{a}$ & $\mathrm{c}$ & c & c & $\mathrm{x}$ & $\mathbf{x}$ & $\mathrm{x}$ & $\mathrm{x}$ \\
\hline Recognition Code & a & c & $\mathrm{y}$ & $\mathrm{a}$ & c & $\mathrm{y}$ & a & $\mathrm{c}$ & $\mathrm{x}$ & $y$ \\
\hline $\begin{array}{l}\text { Correct Recognitions } \\
\text { Repeat Item }\end{array}$ & + & & & & + & & & & + & \\
\hline $\begin{array}{l}\text { Correct Recognitions } \\
\text { Missing Item }\end{array}$ & & + & + & + & & + & + & + & & + \\
\hline
\end{tabular}

The following task would seem to meet these requirements. The procedure involves three steps. The first step is a study trial on a paired-associate list. The second step is a test trial in which all but one of the stimuli are presented for recall of the associated responses. The third and crucial test is to present either one of these stimuli again or to present the missing stimulus. The $S$ is required to first indicate whether the test stimulus has been presented before on the recall trial or whether it is the missing stimulus, and then to attempt recall of the response associated with that stimulus.

Various measures are available, and they have different theoretical implications. In order to examine these implications, it is necessary to consider a simplified list composed of two pairs, A-B and C-D. The procedure requires $A-B$ and $C-B$ to be presented on a study trial and $A$ to be presented on a recall test. The final test involves presentation of either $\mathrm{A}$ or $\mathrm{C}$.

Assume that on the study trial $\mathrm{A}$ is encoded as a and $\mathrm{C}$ as $\mathrm{c}$. On the recall test, $\mathrm{A}$ may be coded as a, c, or $\mathrm{X}$, a different code not used on the study trial. The content of the codes is not of any concern, merely its functional properties. The two different kinds of test stimuli must be considered separately. Table 1 presents the possible events that can occur when $A$ is re-presented on the test trial. There are now four possible codes that could occur: a, c, x, or $y$, another new code not used on either study or test.

The critical information in Table 1 is the correspondence of the code generated at recall and on the test trial. A correct recognition will occur whenever the recall code and the test code are identical, that is, whenever the $S$ codes the stimulus the same on both recall tests and at re-presentation. This will be true whether or not the code is the same as that used on the study trial. Thus, the frequency of correct recognitions on repeated stimuli represents encoding consistency (Martin, 1968), that is, the generation of a stable stimulus code. Variables which affect recognition of repeated items have their effect on encoding variability.

Consider next the events that occur with a $\mathrm{C}$ test, i.e., a test with the missing stimulus. The same possible encodings exist as with repeated stimuli, namely, a, c, x, or $\mathrm{y}$. Note, however, that for this kind of test, the correct recognition of the missing item will occur when the code generated to $\mathrm{C}$ is different or discriminable from that generated to A on the recall test. Thus, the proportion of correct recognitions on missing items indicates the extent of discriminative coding. Variables which affect this measure thus affect code confusion.

The results of a simple experiment may illustrate the use of the procedure. In this experiment, Ss were given the three-stage procedure described above on 40 different lists, 20 on each of 2 consecutive days.

\section{METHOD}

\section{Materials}

The stimuli for one group of Ss were six one-syllable nouns. Three other groups learned lists in which the stimuli were consonant trigrams. In both cases, response terms were two-syllable adjectives. The lists were structured after a pattern introduced by Bruce and Murdock (1968). There were two sets of two pairs in which stimuli within a set were similar, but dissimilar to stimuli in the other sets. The stimuli of the remaining two pairs were dissimilar to one another and dissimilar to the other four stimuli as well. For the words, the high-similarity stimuli rhymed. For the consonant trigrams, highly similar stimuli shared two letters. Within each list, the location of the discriminative letter differed in the two similar sets. Response terms were all unrelated to the stimuli and to one another. No attempt was made to restrict the range of association values with consonant trigrams, although highly meaningful sequences were avoided. Likewise, no attempt was made to control the attributes of the word stimuli.

\section{Procedure}

The sequence for a $S$ on a given list was as follows: a study trial or trials in which each pair was presented separately, a test trial on which five of the six stimuli were presented one at a time for recall, and, finally, presentation of either one of these five stimuli or the remaining stimulus. On this final test, $S$ was required to first rate the stimulus on a six-category confidence scale ranging from 1 (certain it was presented before) to 6 (certain it was the missing item). He then had to attempt recall of the response to this stimulus.

Presentation rate on study trials was 2 sec. Recall was S-paced. Following the last item on the recall, an asterisk appeared followed by the final test stimulus. No time limit was imposed on $\mathrm{S}$ in making his recognition decision or recalling the response.

The group having word stimuli had two study trials prior to the start of testing. The three groups having consonant trigram stimuli had one, two, or five study trials preceding the test sequence. Presentation order varied on multiple study trials and was also different on the test trial. There was no intertrial interval.

\section{Design}

The 20 lists used on each day provided one replication of a 2 by 2 by 5 factorial arrangement of missing item vs repeat item, high vs low similarity of the test item, and position of the tes item (or its paired partner) on the recall test. The order of the 20 conditions was random. Each $\mathrm{S}$ in addition practiced on four lists of nonsense shapes and numbers prior to starting on the test stimuli. A total of $78 \mathrm{Ss}$ participated in the experiment. There were $20 \mathrm{Ss}$ each in the one- and two-trial consonant trigram conditions, $17 \mathrm{Ss}$ in the five-trial consonant trigram group, and $21 \mathrm{Ss}$ in the word group. Groups were tested consecutively. All Ss were introductory psychology students who participated as part of a course requirement.

\section{RESULTS}

\section{Standard Recall}

The results of recall performance on the five items presented prior to the test stimulus showed consistent 
differences between the low- and high-similarity items for the consonant trigram stimuli. After one trial, the proportion of correct recalls was .18 for low-similarity pairs and .16 for high-similarity pairs, $t(19)=.24$. After two trials, recall proportions were .32 for low-similarity pairs and .26 for high-similarity pairs, $t(19)=3.07$, $\mathrm{p}<.01$. After five trials, the low-similarity pairs produced a proportion of .57 correct recalls, while the value for high-similarity pairs was $.49, \mathrm{t}(16)=2.93$, $\mathrm{p}<.01$. For the word stimuli, recall proportion was 67 to both high- and low-similarity stimuli. The standard errors for these comparisons were $1.85,1.79$, and 2.37 for the one-, two-, and five-trial groups, respectively.

\section{Recognition}

The results of real interest are those obtained on the test stimulus. These results will be discussed in some detail. Figure 1 presents two overall measures of recognition performance. The upper half shows the mean confidence rating on repeated and missing items. In this figure, lower ratings indicate greater accuracy for the repeated items, while higher ratings indicate better performance for the missing items. The lower half of the figure presents proportion of recognition errors, i.e., for repeated items the proportion of 4, 5, 6 ratings and for missing items the proportion of $1,2,3$ ratings. The functions are for the consonant trigram stimuli, while the isolated points are for the word stimuli. The two measures lead to identical conclusions.

The first result of interest is performance on the repeat items as shown in Fig. 1 by the solid data points. The theoretical analysis identifies recognition accuracy with encoding stability. There are two findings which are relevant. First, for consonant trigram stimuli, similarity had only a small effect, but it was the high-similarity stimuli which were better recognized. For the more sensitive rating measure, 40 of the 57 Ss performed better on high-similarity tests, $p<.05$ by the sign test. The result may be rationalized by considering letter selection to be the primary means by which $S$ attains a consistent code. The presence of redundant letters in HS stimuli could lead Ss to select more efficiently in these stimuli. To state it in a somewhat different way, $S$ has less to remember in order to recognize an HS stimulus than he does for an LS stimulus. Secondly, at the two-trial comparison point, words were recognized more accurately than either HS or LS consonant trigrams. For low similarity, $F(1,39)=20.66, p<.01$, and for high similarity, $F(1,39)=8.64, p<.025$ for the mean rating measure. Error variances for these comparisons were 32.55 and 35.73 , respectively. Following Martin's (1968) contention that the perception of words as units leads to more consistent coding, this result is confirmatory.

Both results are obviously indirect and depend not only on assumptions concerning the meaning of the repeat item recognition, but also on speculations concerning the effects of various variables on underlying

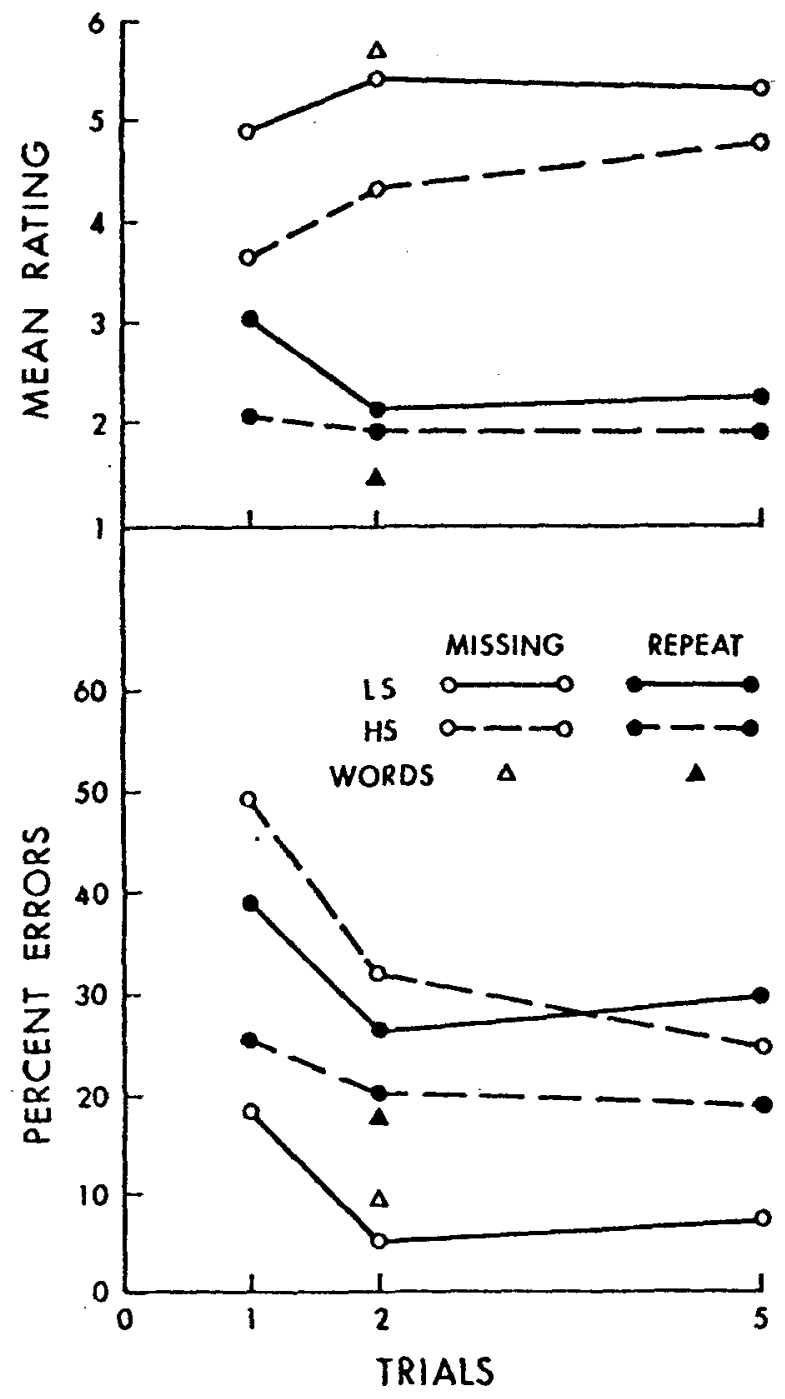

Fig. 1. Mean confidence ratings and recognition errors for missing and repeated stimuli.

process. The point is that the result is consistent with the total set of assumptions.

With respect to cue confusion, the critical measure is performance on the missing item, shown in Fig. 1 as open data points. With consonant trigrams, high-similarity stimuli produced more recognition errors than the low-similarity stimuli, $F(1,54)=129.49$, $p<.01$, error mean square 1.29 . Similarity also had a reliable effect on ratings, as 51 of the $57 \mathrm{Ss}$ performed more poorly on the high similarity, $p<.01$ by the sign test. Of some interest is the trend over trials for these stimuli. Over 20 years ago, Gibson (1940) proposed that cue confusion (which she called generalization) increased then decreased during learning. Several years later, Murdock (1958) questioned this assertion, but the difference of opinion was never satisfactorily resolved. It now appears that Murdock was correct. If the difference between recognition of HS and LS items represents differences in cue confusion, the trend was for maximal confusion to occur on the initial trial and decrease 


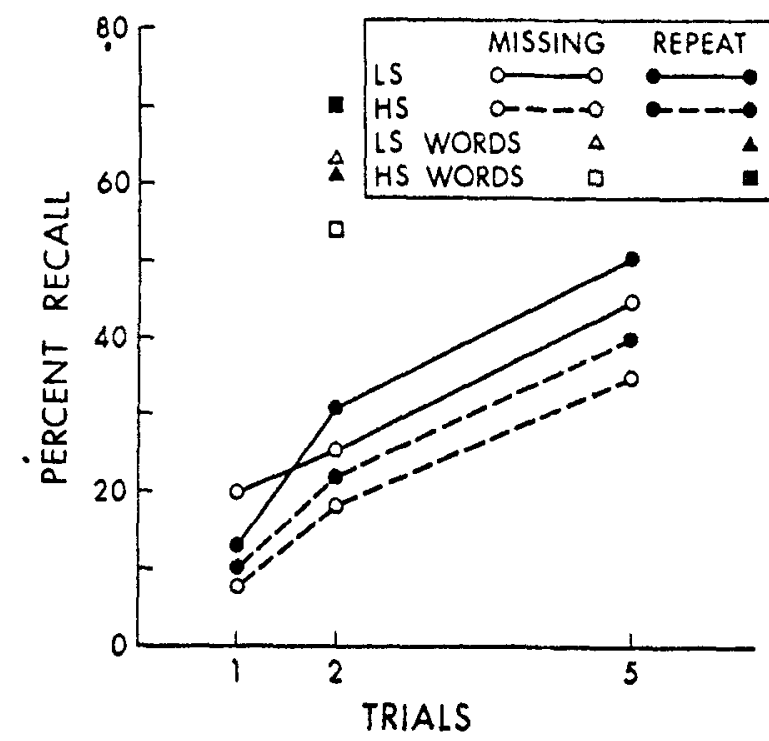

Fig. 2. Percent recall of responses to missing and repeated stimuli.

thereafter. The interaction of trials with similarity was significant, $F(2,54)=5.48, p<.01$, error mean square 1.29.

A second aspect of the effects over trials is the relationship between cue confusion measures and overall performance on the standard recall trial. A comparison of the data of Fig. 1 with the standard recall results shows that the effect of similarity on standard recall increased with practice, while the effects of similarity on recognition of the missing item decreased with practice. This negative covariation is also reflected by an analysis of individual Ss. On the standard recall test, the percentage of Ss who showed better recall to low- than high-similarity stimuli increased with practice from $50 \%$ after one trial to $67 \%$ after two trials and $88 \%$ after five trials. However, the number of Ss who exhibited poorer recognition of high- than of low-similarity stimuli decreased over trials from $100 \%$ after one trial to $90 \%$ after two trials and $76 \%$ after five trials. It would appear then that overall interference in recall increased with practice, although cue confusion was decreasing. Again, Murdock's (1958) argument is vindicated.

There were no effects of similarity with words as stimuli. The reason for the null effect is not apparent, although other studies (Bruce \& Murdock, 1968) have not produced substantial interference due to similarity using the single-trial procedure.

\section{Test Item Recall}

Overall recall to repeated and missing stimuli from high and low similarity is shown in Fig. 2. The functions are shown for trigrams and the isolated points are for words.

Considering first the trigram stimuli, the results were not basically different from those obtained in standard recall. Recall improved with trials, $F(2,136)=24.91$, $p<.01$, error mean square 8.89 . In contrast to the standard recall data, the effect of similarity was virtually uniform over trials, i.e., the interaction between these variables was not significant, $\mathrm{F}<1.00$, error mean square 1.61. There was also no difference in the effectiveness of missing stimuli and repeated stimuli as recall cues, $\mathrm{F}<1.00$, nor did this variable interact with any others.

The pattern of results on the words is complex. Their overall superiority over consonant trigram stimuli is obvious and expected. An unusual aspect of these data is the apparent differential effect of similarity on repeated and missing items. With repeated stimuli, recall was better with high similarity, while for missing stimuli recall was better with low similarity. The interaction was significant, $F(1,60)=9.17, p<.005$, error mean square 1.81 . There is no readily apparent reason why phonemic similarity should be helpful on the second test of an item. Nor is the superiority of low-similarity stimuli on missing item tests consistent with the standard recall data where no similarity effect appeared. The result thus remains a puzzle.

\section{Conditional Recall}

It has become common practice wherever two performance measures are taken on the same task to conditionalize one measure on another. The test trial provides a measure of recognition and recall, and thus would appear to be suitable for such analyses. The interpretation of conditional performance measures is not without ambiguity because of item and $\mathrm{S}$ selection biases (Runquist, in press), but the discussion of these difficulties will be postponed until the results have been presented.

The most relevant question theoretically is whether recall depends upon successful recognition performance. In order to examine this relationship, stimuli were first categorized as to whether they were correctly recognized or not, and the proportion of correct recalls was determined for each category. Missing and repeated stimuli were considered separately, as were high- and low-similarity stimuli within this category. The analysis was performed only on consonant trigrams, as there were too few recognition errors with word stimuli to provide a stable comparison. The groups having varying numbers of trials were also combined to increase the stability of the results. The conditional recall measures are presented in Table 2. Also shown in the table is the proportion of correct recognitions.

The results are quite consistent and interpretable. For repeated stimuli, a correct recognition should indicate identical coding on the two presentations. The first result of note is that there was virtually no successful recall when the stimulus was not correctly recognized. Relevant to Martin's (1968) theory, one could conclude that recall is nil unless a stable code has been established. The second finding is that recall to low-similarity stimuli exceeded that to high-similarity stimuli. Despite equal 
"code stability," there was still interference produced by similarity. This result provides additional support for the contention that tests on repeated items are independent of cue confusion. If the sample of recognized items contains both discriminated and nondiscriminated items, the effect of similarity would be as it appeared here.

The results for the missing stimuli are equally in accord with a coding interpretation. When stimuli were correctly identified, and presumably correctly discriminated, there was no difference in recall to highand low-similarity stimuli. On the other hand, when stimuli were not correctly discriminated, recall to low-similarity stimuli was approximately twice as great.

This latter finding provides a mild puzzle, but is, at least, resolvable on an ad hoc basis. If all interference is due to cue confusion, one might expect no difference in recall to low- and high-similarity stimuli for nonrecognized missing items as well as for correctly recognized items. The effect could, however, represent associative interference mediated by the similarity between stimuli.

Despite the apparent validity of these conclusions, the presence of possible item and $\mathrm{S}$ sampling biases suggests that the data be approached with some caution. Unfortunately, every difference observed in the present data can be accounted for by assuming that larger samples of items include more difficult items, and hence differences will always be biased toward lower recall for the category containing more items. Thus, for repeated stimuli, differences on recognized stimuli would be enhanced, while for missing stimuli, differences would be reduced on recognized items and enhanced for nonrecognized stimuli. Since the extent of the bias cannot be estimated, the extent to which the pattern of results in Table 2 represents genuine effects of coding processes also cannot be determined with certainty.

A second way of looking at conditional measures would be to examine recognition of recalled and nonrecalled items. It has been argued (Underwood \& Freund, 1968) that Ss use response terms to differentiate stimuli. Since the response term is not available on the test trial unless $S$ supplies it himself, we might expect discriminative encoding to be better when responses are recallable. In order to test this hypothesis, the proportion of correctly identified missing stimuli was examined for those stimuli to which correct recall occurred and for those to which it did not. For recalled items, this proportion was .91 for LS and .83 for HS items. For nonrecalled items, the proportions were .73 for LS, but only .44 for HS items. In short, stimuli were much better discriminated when the response was recallable. When $S$ was not able to recall the response, similarity among stimuli had a greater effect.

On repeated stimuli, the trend was reversed. Again, correct stimulus identification was superior when recall was successful; for both HS and LS items, recognition proportion was .96 . When recall failed, Ss identified .72 of the HS stimuli and .56 of the LS stimuli. Stimulus
Table 2

Conditional Recall Proportions

\begin{tabular}{lccc}
\hline & $\begin{array}{c}\text { Recog- } \\
\text { nized } \\
\text { Stimuli }\end{array}$ & $\begin{array}{c}\text { Nonrecog- } \\
\text { nized } \\
\text { Stimuli }\end{array}$ & $\begin{array}{c}\text { Correct } \\
\text { Recog- } \\
\text { nitions }\end{array}$ \\
\hline Repeated Stimuli & & & \\
$\quad$ Low Similarity & .43 & .04 & .68 \\
$\begin{array}{l}\text { High Similarity } \\
\text { Missing Stimuli }\end{array}$ & .31 & .05 & .78 \\
$\quad$ Low Similarity & .27 & .25 & .90 \\
$\quad$ High Similarity & .30 & .12 & .66 \\
\hline
\end{tabular}

coding was less variable when the response was recallable. When $\mathrm{S}$ was not able to recall the response, variability was greater with low-similarity stimuli.

If one takes these results at face value, it could be concluded that the recall of the response term aided in both the discriminative and stable encoding of the stimulus. There is little evidence as to how this facilitation occurs, but some possibilities might be suggested. One way is that on the test trial, $S$ recalls the response then matches this response with previous recalls. If he obtains a match, he considers the stimulus repeated; if no match is obtained, he identifies the item as the missing item. One piece of evidence consistent with this interpretation is the fact that there is an overall bias to classify an item as the missing one. To put it another way, recognition performance is better on missing items than on repeated ones. Since a failure to find a match can rresult from the failure to recall the response to the first occurrence of the stimulus, or the forgetting of that response, the overall frequency of "missing" responses would be greater.

The second hypothesis is more theoretically interesting. The S may not discriminatively encode the stimulus term at recall, but actually use the response term to make the discrimination by what is essentially a backward association procedure. For example, we present JOX. The $S$ retrieves two associations, say "tiger" and "house." In order to determine which one is correct, he now recalls that "tiger went with Q" while "house doesn't match the discriminative letter." While there is nothing in the present data to indicate the operation of this process, it remains a plausible hypothesis. Of course, strong conclusions based on conditional response measures are quite dangerous. In addition to the item/S selection bias discussed earlier, the causal direction of inference is always ambiguous because of the essentially correlational nature of these measures. That is, are recall differences caused by recognition differences, or are recognition differences caused by recall differences? The results of this experiment may be considered both ways with theoretically reasonable interpretations in either case.

\section{Further Analyses}

Another means of evaluating the theoretical hypothesis concerning the meaning of recognition errors makes use of the correspondence of responses recalled 


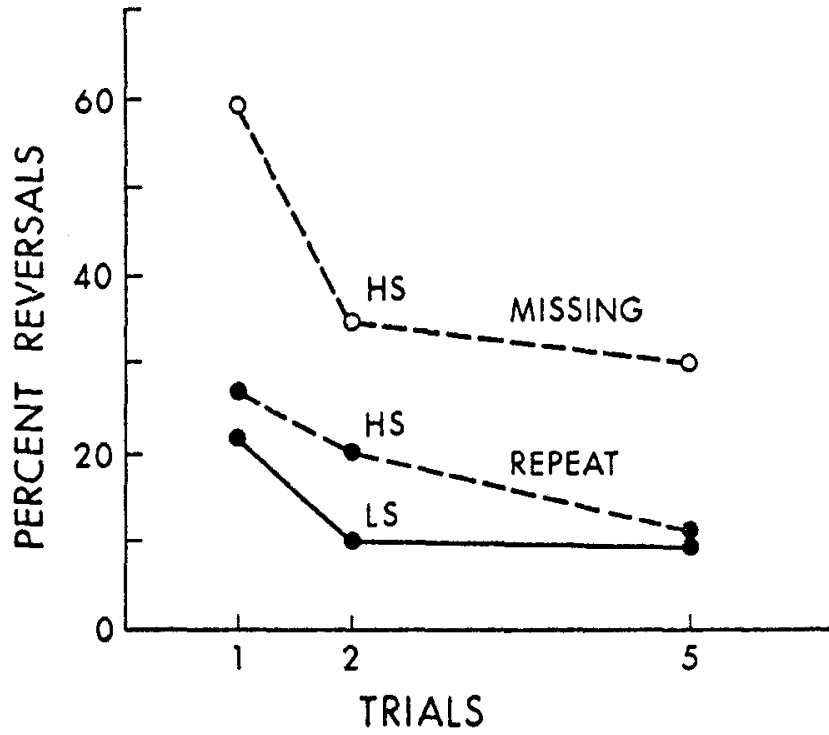

Fig. 3. Percent of responses changed from recall test to recognition test on missing and repeated items.

on the recognition-test trial to those recalled on the standard recall.

Consider first repeated stimuli. A correct recognition should indicate that the stimulus was encoded the same on both presentations. If this is so, the response recalled should also be the same, i.e., the $S$ should not switch responses. If he does, it must mean that two associations exist to the same stimulus code. In this analysis, items in which a response was given to a stimulus on both standard recall and test presentations were considered, given that he correctly recognized the stimulus as repeated. The proportion of times that he changed his response on the test trial was determined irrespective of correctness. These results are shown as the lower set of functions in Fig. 3. The proportion of reversals clearly decreased over trials. Differences between HS and LS items were generally small, but maximal after two trials.

The same analysis was performed on missing items, except that here only those items which $S$ failed to recognize were considered. If a recognition error means that $S$ has encoded the test stimulus the same as one of the stimuli presented on the standard recall, he should also recall the same response. The upper curve in Fig. 3 shows the proportion of reversals for HS items. There were too few LS items to determine a function for those items. Notable is the fact that reversals decreased over trials and were considerably more frequent than reversals to correctly recognized repeated stimuli.

This analysis is rather puzzling. If it truly indicates associative interference, i.e., two responses associated with the same code, one could conclude that associative competition is a minimal factor in producing interference. Yet such a conclusion is not consistent with other results. It will be recalled that overall interference, as indicated by recall performance, increased over trials, while cue confusion, as indicated by recognition of missing items, decreased over trials. If associative interference is to account for the interference in recall, it should show an increase over trials.

This anomaly brings up an important point with respect to analyses of this kind. They assume that each recognition response is exactly determined. That is, when $\mathrm{S}$ identifies a stimulus as a repeated stimulus, he has not, in fact, discriminatively coded that particular stimulus from others. It is likely, however, that some recognition responses are not interpretable in this unambiguous fashion. For example, the $\mathrm{S}$ may forget one or more of the stimulus codes he generated on the standard recall tests, including the one to the critical stimulus. Thus, he is unable to find an appropriate match and hence classifies the stimulus as "missing." The overall higher frequency of "missing" classifications would seem to support this. The use of the confidence rating scale gives some hope of resolving this problem, by considering for the repeated items only those where $S$ was certain it was repeated and tabulating response reversals. The proportions were .28 and .31 for HS and LS items after one trial, .20 and .06 after two trials, and .06 and .10 after five trials. Again the only difference is after two trials. It would seem that one cannot account for the difference in recall between high- and low-similarity items after five trials by associative interference. The only means of resolving this apparent contradiction is to assume that $\mathrm{S}$ operates on the basis of recalled responses rather than stimulus codes.

\section{DISCUSSION}

Two discussable points emerge from these results, one methodological and one theoretical. The methodological point is whether the missing item and repeat item tests do indeed reflect stimulus discrimination and encoding variability, respectively. The theoretical point is whether these particular results tell us anything about the processes involved in interference.

With respect to the first point, all of the data are consistent with the cue confusion-variability hypothesis. The effects of similarity on the recognition of missing items and the effects of meaningfulness (i.e., words vs consonant trigrams) on the recognition of repeated items provides the strongest evidence for the hypothesis. Whether one can interpret individual responses in the manner suggested by the model presented in the introduction is another question, however. There are problems with conditional measures and other indices which treat responses in a highly individualized manner. Moreover, it is virtually necessary to assume that no processes other than those specified by the model operate to determine a specific response. Experience with explicit models should by now produce sufficient caution in making such deterministic assumptions.

Nevertheless, the present data suggest that at a gross level, the missing item and repeat item recognition tests can be used to provide measures of cue confusion and 
encoding variability which are independent of recall performance and yet do not impose task demands other than those involved in retrieval.

The theoretical issue is more difficult to resolve. Nevertheless, some problems may be brought into focus by considering the overall pattern of results. The issue is joined by the lack of correlation between various measures of performance and the overall effects of similarity on recall. If these results are accepted at face value, we are left in the position that none of the postulated processes seem to account for similarity effect, and hence interference.

Implicit in the operational formulations of cue confusion, encoding variability, and associative competition is a particular conceptual model for recall of paired associates. It is assumed, not only here but in most contemporary theory, that recall proceeds in two discrete and temporally ordered stages. The $\mathrm{S}$ first encodes the stimulus, then in some manner uses the stimulus code to retrieve the response term.

Also, it is implied by this assumption that the source of interference is in the retrieval of the response term. It is these assumptions that may be challenged. Similarity can have effects in recognition or multiple choice paradigms where retrieval is not a problem (Muir, 1966). Suppose, then, that interference results primarily from the simultaneous activation of several potential response terms plus the failure to discriminate the correct response from incorrect ones. Cue discrimination does not determine which responses are activated, but is involved primarily in aiding the discrimination of the correct association. The fact that there are other variables (e.g., recency) which aid discrimination would tend to reduce the correlation between cue confusion measures and interference. The theory also suggests that the causal direction of the correlation between recognition and recall is backwards, i.e., recognition is dependent on successful recall.

Obviously, these notions are very speculative. However, the data presented here point out that the common conception of a sequentially ordered retrieval process beginning with stimulus coding may be incorrect, and there are other reasonable possibilities for accounting for interference.

\section{REFERENCES}

Bruce, D., \& Murdock, B. B. Acoustic similarity effects on memory for paired associates. Journal of Verbal Learning \& Verbal Behavior, 1968, 7, 627-631.

Ellis, H. C., \& Muller, B. Transfer in perceptual learning following predifferentiation. Journal of Experimental Psychology, 1964, 68, 388-395.

Gibson, E. J. A systematic application of the concepts of generalization and differentiation to verbal learning. Psychological Review, 1940, 47, 196-229.

Martin, E. Relation between stimulus recognition and paired associate learning. Journal of Experimental Psychology, 1967, 74, 500-505.

Martin, E. B. Stimulus meaningfulness and paired associate transfer: An encoding variability hypothesis. Psychological Review, 1968, 75, 421-440.

Muir, W. R. Measurement of similarity of verbal material by a paired comparison procedure. PhD dissertation, University of Alberta, 1966.

Murdock, B. B. Intralist generalization in paired associate learning. Psychological Review, 1958, 65, 306-314.

Rothkopf, E. Z. A measure of stimulus similarity and errors in some paired associate learning tasks. Journal of Experimental Psychology, 1957, 53, 94-101.

Runquist, W, N. Conditional recall in memory experiments. American Journal of Psychology, in press.

Runquist, W. N., \& Evans, A. Stimulus recognition and associate coding. Journal of Experimental Psychology, 1972, 95, 242-244.

Underwood, B. J., \& Freund, J. S. Transfer of stimulus discrimination: Can $R$ terms be used to differentiate stimulus terms? Journal of Verbal Learning \& Verbal Behavior, 1968, 7, 825-830.

(Received for publication October 4, 1973; revision received December 7,1973 .) 